\title{
ON DIRECT SUMS OF REFLEXIVE OPERATORS
}

\author{
AVRAHAM FEINTUCH
}

Abstract. Let $A_{1}$ and $A_{2}$ be reflexive operators on a Hilbert space $H$. If $A_{2}$ is algebraic then $A_{1} \oplus A_{2}$ is reflexive.

1. Introduction. Let $H$ be a complex Hilbert space and $B(H)$ the algebra of all bounded linear operators on $H$. For $A \in B(H)$, Lat $A$ will denote the set

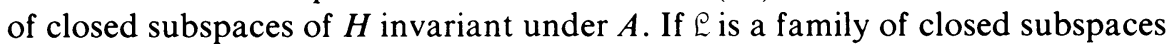
in $H$, then $\mathrm{Alg} \mathcal{L}$ will denote the (weakly closed) algebra of operators which leave invariant every member of $e . \mho(A)$ will denote the weak closure of the algebra of polynomials in $A$. $(A)^{\prime}$ will denote the commutant of $A$.

Definition. $A$ is reflexive if $\mathcal{Q}(A)=\mathrm{Alg}$ Lat $A$. Reflexive operators have been studied by various authors ([1], [2], [4]) and the following question was raised in [3]: If $A_{1}$ and $A_{2}$ are reflexive, is $A_{1} \oplus A_{2}$ reflexive?

In this note it is shown that if $A_{2}$ is algebraic then $A_{1} \oplus A_{2}$ is reflexive.

2. Preliminaries. $H^{(k)}$ will denote the direct sum of $k$ copies of $H$, and for $A \in B(H), A^{(k)}$ will be the $k$-fold copy of $A$ acting on $H^{(k)}$, i.e. if $\left\langle x_{1}, \ldots, x_{k}\right\rangle \in H^{(k)}$, then $A^{(k)}\left\langle x_{1}, \ldots, x_{k}\right\rangle=\left\langle A x_{1}, \ldots, A x_{k}\right\rangle$.

LEMMA 1. If for every positive integer $n \geqq 1$, Lat $A^{(n)} \subseteq$ Lat $B^{(n)}$, then $B \in$ थ $(A)$.

This lemma is quite well known and is a standard tool used in the study of reflexive operators.

3. Algebraic operators. In this section we obtain some results for algebraic operators. These will be used in the proof of the main theorem.

Theorem 1. Let $A$ be an algebraic operator. Then $\mathscr{Q l}(A)=(\mathrm{Alg}) A) \cap(A)^{\prime}$.

Proof. Suppose $B \in($ Alg Lat $A) \cap(A)^{\prime}$. We will show that for all positive integers $n$, Lat $A^{(n)} \subseteq$ Lat $B^{(n)}$. The result will then follow by Lemma 1 .

Suppose $\Re \in$ Lat $A^{(n)}$. Since every invariant subspace of $A^{(n)}$ is a span of cyclic subspaces, we can assume $\Re$ is a cyclic subspace. Since $A^{(n)}$ is algebraic, $\mathfrak{R}$ is finite dimensional.

For $1 \leqq i \leqq n$, let $\pi_{i}(\Re)$ be the projection of ฯ on the $i$ th coordinate space and let $\pi(\Re)=\bigvee_{i=1}^{m} \pi_{i}(\Re)$. Then $\pi(\Re)$ is finite dimensional and is clearly in Lat $A$ (since $\pi_{i}(\mathfrak{N})$ is, for each $i$ ). Therefore, $\pi(\mathfrak{N}) \in$ Lat $B$.

Denote the restrictions of $A$ and $B$ to $\pi(\Re)$ by $\hat{A}$ and $\hat{B}$ respectively. Then

Received by the editors January 6, 1975.

AMS (MOS) subject classifications (1970) Primary 47B10.

Key words and phrases. Reflexive operator, invariant subspaces, algebraic operator, commutant. 
$\hat{B} \in($ Alg Lat $\hat{A}) \cap(\hat{A})^{\prime}$. Thus, by [2], there is a polynomial $p$ such that $B x=p(A) x$ for all $x \in \pi(\mathscr{T})$.

Thus if $\left\langle x_{1}, \ldots, x_{n}\right\rangle$ is an arbitrary vector in $\Re, x_{i} \in \pi(\Re)$ for $1 \leqq i \leqq n$. Then $B x_{i}=p(A) x_{i}$ and $B^{(n)}\left\langle x_{1}, \ldots, x_{n}\right\rangle=\left\langle p(A) x_{1}, \ldots, p(A) x_{n}\right\rangle$. This completes the proof.

Corollary 1. Let $A$ and $B$ be reflexive algebraic operators. Then $A \oplus B$ is reflexive.

Proof. Suppose Lat $A \oplus B \subseteq$ Lat $T$. Since $\{\langle x, 0\rangle: x \in H\}$ and $\{\langle 0, x\rangle: x$ $\in H\} \quad$ are in Lat $A \oplus B$, it follows that $T=E \oplus F$ with Lat $A$ $\subset$ Lat $E$ and Lat $B \subset$ Lat $F$. Since $A$ and $B$ are reflexive, $A E=E A$ and $F B$ $=B F$.

Now $A \oplus B$ is algebraic and $E \oplus F \in[\operatorname{Alg} \operatorname{Lat}(A \oplus B)] \cap(A \oplus B)^{\prime}$, so we can apply Theorem 1 .

4. The main result. In this section we show that if $A_{1}$ is algebraic reflexive and $A_{2}$ is reflexive then $A_{1} \oplus A_{2}$ is reflexive.

We will first prove a special case and the above result will easily follow.

TheOREM 2. Suppose $A$ is nilpotent reflexive and $B$ is reflexive. Then $A \oplus B$ is reflexive.

Proof. Suppose $T \in \operatorname{Alg} \operatorname{Lat}(A \oplus B)$. The argument used in Corollary 1 shows that $T=E \oplus F$ with $E \in \mathcal{Q}(A)$ and $F \in \mathcal{Q}(B)$. Thus $E \oplus F \in(A$ $\oplus B)^{\prime}$ and Lat $A \oplus B \subseteq$ Lat $E \oplus F$.

It will be shown that $\operatorname{Lat}(A \oplus B)^{(n)} \subseteq \operatorname{Lat}(E \oplus F)^{(n)}$. It suffices to show this for $n=2$. For then the same hypotheses apply to $(A \oplus B)^{(2)}$. We can then apply the same argument to show that $\operatorname{Lat}(A \oplus B)^{(4)} \subseteq \operatorname{Lat}(E \oplus F)^{(4)}$. If we continue this process we obtain that $\operatorname{Lat}(A \oplus B)^{\left(2^{n}\right)} \subseteq \operatorname{Lat}(E \oplus F)^{\left(2^{n}\right)}$. But this implies Lat $(A \oplus B)^{(k)} \subseteq \operatorname{Lat}(E \oplus F)^{(k)}$ for all integers $k \geqq 1$.

We identify $\operatorname{Lat}(A \oplus B)^{(2)}$ with $\operatorname{Lat}\left(A^{(2)} \oplus B^{(2)}\right)$, and will show

$$
\operatorname{Lat}\left(A^{(2)} \oplus B^{(2)}\right) \subseteq \operatorname{Lat}\left(E^{(2)} \oplus F^{(2)}\right)
$$

Suppose $\mathbb{O} \in \operatorname{Lat}\left(A^{(2)} \oplus B^{(2)}\right)$. We consider two cases:

Case (i). $\because \pi$ does not contain a vector of the form $\langle 0, y\rangle$. Here 0 is the zero vector in $H^{(2)}$ and $y \in H^{(2)}$. Then (by a well-known argument) $0 \pi$ is the graph of some closed operator $T$ with domain $\mathcal{Q}(T)$ in $H^{(2)} ; \mathcal{N}=\{\langle x, T x\rangle: x$ $\in \mathscr{Q}(T)\}$. Then $\mathscr{N} \in \operatorname{Lat}\left(A^{(2)} \oplus B^{(2)}\right)$ implies $B^{(2)} T x=T A^{(2)} x, x \in \mathscr{Q}(T)$. Suppose the index of $A$ is $n$. Then $A^{(2) n}=0$. This implies that $B^{(2) n} T x$ $=0$ for $x \in \mathfrak{Q}(T)$. If $\Re(L)$ is the null space of the operator $L$, then

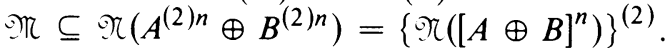

Denote the restrictions of $A \oplus B$ and $E \oplus F$ to $\Re\left([A \oplus B]^{n}\right)$ by $S_{1}$ and $S_{2}$ respectively. Then $S_{1}$ is nilpotent, and $S_{2} \in\left(\right.$ Alg Lat $\left.S_{1}\right) \cap\left(S_{1}\right)^{\prime}$. By Theorem $1, S_{2} \in \mathcal{U}\left(S_{1}\right)$. This clearly implies that $\Re \in \operatorname{Lat}\left(E^{(2)} \oplus F^{(2)}\right)$.

Case (ii). ơ contains a vector of the form $\langle 0, y\rangle$ with $y \neq 0$. Let $\Re$

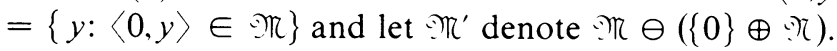

Then $\mathcal{R}^{\prime}$ is the graph of some closed operator $T$; $\mathbb{O}^{\prime}=\{\langle x, T x\rangle: x$ $\in \mathscr{Q}(T)\}$. Also, 


$$
\begin{aligned}
A^{(2)} \oplus B^{(2)}\langle x, T x\rangle & =\left\langle A^{(2)} x, B^{(2)} T x\right\rangle \\
& =\left\langle A^{(2)} x, T A^{(2)} x\right\rangle+\left\langle 0,\left(B^{(2)} T-T A^{(2)}\right) x\right\rangle,
\end{aligned}
$$

where the last element is in $\{0\} \oplus \Re$.

We can assume that $\Re$ is a cyclic invariant subspace of $A^{(2)} \oplus B^{(2)}$. Thus there exist vectors $x_{1}, x_{2}, y_{1}, y_{2}$ such that

$$
\mathscr{R}=\bigvee_{i=0}^{\infty}\left\langle A^{i} x_{1}, A^{i} x_{2}, B^{i} y_{1}, B^{i} y_{2}\right\rangle .
$$

Then if the index of $A$ on $\mathscr{D}(T)$ is $n, \mathscr{R}=\bigvee_{i=n}^{\infty}\left\langle B^{i} y_{1}, B^{i} y_{2}\right\rangle$ and $\mathscr{D}(T)$ and $\mathscr{R}(T)$ are finite dimensional. Let $P$ denote the projection on $\Re(T)$. Since $\Re$ $\in$ Lat $B^{(2)}$, it follows that $\Re^{\prime} \in \operatorname{Lat}\left(A^{(2)} \oplus P B^{(2)} P\right)$. This implies that $T A^{(2)}=P B^{(2)} P T$ and therefore $P B^{(2)} P$ is nilpotent of index $n$.

Since $F \in \mathcal{Q}(B)$ and $\Re(T) \oplus \Re \in$ Lat $B^{(2)}$, it is easy to see that $P F^{(2)} P$ $\in \mathcal{Q}\left(P B^{(2)} P\right)$. Thus there exist polynomials $q$ and $r$, both of degree less than $n$, such that $E^{(2)}=q\left(A^{(2)}\right)$ and $P F^{(2)} P=r\left(P B^{(2)} P\right)$.

To complete the proof it is enough to show that $\Re^{\prime} \in \operatorname{Lat}\left(E^{(2)} \oplus P F^{(2)} P\right)$. This is equivalent to showing that $r\left(P B^{(2)} P\right)=q\left(P B^{(2)} P\right)$. Let

$$
\Re_{1}=\bigvee_{i=0}^{\infty}\left\langle A^{i} x_{1}, B^{i} y_{1}\right\rangle, \quad \Re_{2}=\bigvee_{i=0}^{\infty}\left\langle A^{i} x_{2}, B^{i} y_{2}\right\rangle .
$$

Since we identify $\mathscr{N}$ with the subspace $\bigvee_{i=0}^{\infty}\left\langle A^{i} x_{1}, B^{i} y_{1}, A^{i} x_{2}, B^{i} y_{2}\right\rangle$, then $\Re \subset \mathfrak{R}_{1} \oplus \mathfrak{R}_{2}$. Since $A$ is nilpotent, $\mathfrak{N}_{i}=\mathfrak{N}_{i}^{\prime} \oplus\left(\{0\} \oplus \mathfrak{\Re}_{i}\right)$ where $\mathfrak{\Re}_{i}$ $=\bigvee_{k=n}^{\infty} B^{k} y_{i}$, and $\mathfrak{T}_{i}^{\prime}=\left\{\left\langle x, S_{i} x\right\rangle: x \in \mathscr{D}\left(S_{i}\right)\right\}$. Then $\mathscr{D}\left(S_{i}\right)=\bigvee_{k=0}^{n-1} A^{k} x_{i}$ and therefore $\mathscr{D}(T) \subseteq \mathscr{D}\left(S_{1}\right) \oplus \mathscr{D}\left(S_{2}\right)$. Also $\Re \subseteq \Re_{1} \oplus \Re_{2}$. By taking orthogonal complements, we obtain that $\mathcal{R}\left(S_{1}\right) \oplus \Re\left(S_{2}\right) \subseteq \Re(T)$.

Now $\mathfrak{R}_{i} \in$ Lat $A \oplus B$ for each $i$. If $Q_{i}$ is the projection on $\mathcal{R}\left(S_{i}\right)$, it follows that $S_{i} A=Q_{i} B Q_{i} S_{i}, i=1,2$. Thus $Q_{i} B Q_{i}$ is nilpotent and its index on $\Re\left(S_{i}\right)$ is the index of $A$ on $\mathscr{D}\left(S_{i}\right)$. It easily follows that the index of $\left(Q_{1} \oplus Q_{2}\right) B^{(2)}\left(Q_{1} \oplus Q_{2}\right)$ on $\Re\left(S_{1}\right) \oplus \Re\left(S_{2}\right)$ is $n$. Recall that this is the index of $P B^{(2)} P$ on $\Re(T)$.

Since $\mathfrak{R}_{i} \in$ Lat $A \oplus B \subseteq$ Lat $E \oplus F$, and $E=q(A)$, it follows that $Q_{i} F Q_{i}$ $=q\left(Q_{i} B Q_{i}\right)$. Thus

$$
\left[Q_{1} \oplus Q_{2}\right] F^{(2)}\left[Q_{1} \oplus Q_{2}\right]=q\left(\left[Q_{1} \oplus Q_{2}\right] B^{(2)}\left[Q_{1} \oplus Q_{2}\right]\right) .
$$

Now $\mathcal{R}\left(S_{1}\right) \oplus \mathscr{R}\left(S_{2}\right)$ is an invariant subspace of $P B^{(2)} P$. Thus on $\mathscr{R}(T)$ $=\left[\Re\left(S_{1}\right) \oplus \Re\left(S_{2}\right)\right] \oplus \mathcal{L}$,

$$
P B^{(2)} P=\left(\begin{array}{cc}
B_{1} & X \\
0 & B_{2}
\end{array}\right)
$$

and

$$
P F^{(2)} P=r\left(P B^{(2)} P\right)=\left(\begin{array}{cc}
r\left(B_{1}\right) & X^{\prime} \\
0 & r\left(B_{2}\right)
\end{array}\right) .
$$

Thus $r\left(B_{1}\right)=q\left(B_{1}\right)$. If $t=r-q$ then $t\left(B_{1}\right)=0$. But the degrees of $r$ and $q$ 
are less than $n$ and $B_{1}$ is nilpotent of index $n$. This implies that $r=q$. This completes the proof.

Theorem 3. Suppose $A$ is algebraic. If $A$ and $B$ are reflexive, then so is $A \oplus B$.

Proof. Since $A$ is algebraic, $A$ is similar to an operator $A^{\prime}=N_{1} \oplus N_{2} \oplus \cdots$ $\oplus N_{k}$ where each $N_{i}$ is a translated nilpotent operator with all translating scalars distinct. By the argument used in [2] (which holds equally well in the infinite-dimensional case) $A^{\prime}$ is reflexive if and only if $N_{i}$ is reflexive for $1 \leqq i \leqq k$. Since reflexivity is preserved under similarities the result follows by induction.

\section{BIBLIOGRAPHY}

1. J. A. Deddens, Every isometry is reflexive, Proc. Amer. Math. Soc. 28 (1971), 509-512. MR 43 \#3831.

2. J. A. Deddens and P. Fillmore, Reflexive linear transformations, J. Linear Algebra 10 (1975), 89-93.

3. P. Rosenthal, Problems on invariant subspaces and operator algebras, Proc. 1970 Tihany Conf. on Hilbert Space Operators and Operator Algebras: Colloq. Math. Soc. Janos Bolyai 5 (1972).

4. D. Sarason, Invariant subspaces and unstarred operator algebras, Pacific J. Math. 17 (1966), 511-517. MR 33 \#590.

Department of Mathematics, Ben Gurion University of the Negev, Beersheva, Israel 